\title{
Kernel-Bayesian Framework for Object Tracking
}

\author{
Xiaoqin Zhang ${ }^{1}$, Weiming $\mathrm{Hu}^{1}$, Guan $\mathrm{Luo}^{1}$, and Steve Maybank ${ }^{2}$ \\ ${ }^{1}$ National Laboratory of Pattern Recognition, Institute of Automation, Beijing, China \\ \{xqzhang, wmhu, gluo\}@nlpr.ia.ac.cn \\ ${ }^{2}$ School of Computer Science and Information Systems, Birkbeck College, London, UK \\ sjmaybank@dcs.bbk.ac.uk
}

\begin{abstract}
This paper proposes a general Kernel-Bayesian framework for object tracking. In this framework, the kernel based method-mean shift algorithm is embedded into the Bayesian framework seamlessly to provide a heuristic prior information to the state transition model, aiming at effectively alleviating the heavy computational load and avoiding sample degeneracy suffered by the conventional Bayesian trackers. Moreover, the tracked object is characterized by a spatial-constraint MOG (Mixture of Gaussians) based appearance model, which is shown more discriminative than the traditional MOG based appearance model. Meantime, a novel selective updating technique for the appearance model is developed to accommodate the changes in both appearance and illumination. Experimental results demonstrate that, compared with Bayesian and kernel based tracking frameworks, the proposed algorithm is more efficient and effective.
\end{abstract}

\section{Introduction}

Object tracking is an important research topic in computer vision community, because it is the foundation of high level visual problems such as motion analysis and behavior understanding. Recent years have witnessed a great advance in the literature, e.g. snakes model [1], condensation [2], mean shift [3], appearance model [4], probabilistic data association filter [5] and so on.

Generally speaking, most of the tracking algorithms involve two major issues: the algorithm framework and the target representation model. The framework of the existing tracking algorithms can be roughly divided into two categories: deterministic methods and stochastic methods. Deterministic methods usually reduce to an optimization process, which can be typically tackled by an iterative search for the minimization of a similarity cost function. In more detail, there exists two major types of similarity functions: SSD (Sum of Squared Differences) [6] and kernel [3] based cost functions. The SSD based cost function is defined as the summation of squared differences between the current image patch and the template, while the kernel based cost function is defined as the distance between two kernel densities. The deterministic methods are usually computationally efficient but often trap in local minimal. In contrast, the stochastic methods adopt a state space to model the underlying dynamics of the tracking process, and the object tracking is viewed as a Bayesian inference problem, which needs to generate a number of hypotheses to estimate and propagate the posterior distribution of the state. Compared with the deterministic counterparts, the stochastic methods usually perform more robustly, but meantime they suffer a heavy computational load due to the large 
number of hypotheses, especially in a high-dimensional state space which may result in the curse of dimensionality. Recently, some researchers have combined the merits of these two methods to achieve more reliable performances [7 [8]. In [7], random hypotheses are guided by a gradient based deterministic search which is carried out based on the sum of difference between two frames. Zhou [8] et al. propose an adaptive state transition model which is extracted from the information contained in the particles configuration. In essence, these methods are based on the constant illumination assumption, which is hard to be satisfied in practice, and moreover, they are far from a general tracking framework to be extended to other representative models.

The target representation model is also a basic issue to be considered in tracking algorithms. Image patch [6], which takes the set of pixels in the target region as the model representation, is a direct way to model the target, but it loses the discriminative information that is implicit inside the layout of the target. The color histogram [3] provides global statistic information of the target region which is robust to noise, but it is very sensitive to illumination changes. Recently the MOG (Mixture of Gaussians) [4|8 9] based appearance model has received more and more attentions for its following merits: (1) it can model the multi-modal distribution of the appearance; (2) it is easy to capture the changes of the appearance; (3) it possess low computation and storage resources. However, traditional MOG based appearance model considers each pixel independently and with the same level of confidence, which is not reasonable in practice.

In view of the forgoing discussions, we propose a general kernel-Bayesian tracking framework by combining the merits of both deterministic methods and stochastic methods. The main contributions of the proposed tracking approach are summarized as follows:

1. The kernel based method-mean shift algorithm is embedded into the Bayesian framework to give a heuristic prior information to the state transition model, which eases the computational burden and avoids sample degeneracy in the Bayesian tracking framework.

2. The appearance of the target is modeled by a spatial constraint MOG, whose parameters are estimated via an on-line EM algorithm.

3. A novel selective adaptation scheme for updating the appearance model is adopted to reliably capture the changes in appearance and illumination and effectively prevent the model from drifting away.

The rest of this paper is structured as follows. A brief review of kernel based and Bayesian based tracking algorithms are presented in Section 2. The detail of KernelBayesian tracking framework is described in Section 3. A spatial constraint MOG based appearance model and its application in the Kernel-Bayesian framework are discussed in Section 4. Experimental results are presented in Section 5, and Section 6 is devoted to conclusion.

\section{Review of Kernel Based and Bayesian Based Trackers}

In this section, we briefly review the two typical tracking algorithms: kernel based and Bayesian based trackers. 


\subsection{Kernel Based Tracker}

Kernel based tracker tries to find local minima of a similarity measure between the kernel density estimations of the candidate and target images. The most famous kernel based method should be the mean shift algorithm, which firstly appeared in [10] as the gradient estimation of a density function, and was introduced for visual tracking by Comaniciu [3] in 2000.

Mean shift is a non-parameter mode seeking technique that shifts each data point to the average of data points in its neighborhood [10]. Let $A$ be a finite set embedded in an $n$-dimensional space $X$, the mean shift vector of $x$ is defined as follows,

$$
m s=\frac{\sum_{a} K(a-x) w(a) a}{\sum_{a} K(a-x) w(a)}-x, a \in A, x \in X
$$

where $K$ is a kernel function and $w$ is a weight function. Mean shift algorithm works by iteratively shifting the data to the direction of mean shift vector until its convergence.

In the mean shift based tracking algorithm, the convergence property is described by a Bhattacharyya coefficient [3], which reflects the similarity between the target and candidate kernel densities.

\subsection{Bayesian Based Tracker}

Another popular way is to view tracking as an on-line Bayesian inference process for estimating the unknown state $s_{t}$ at time $t$ from a sequential observations $o_{1: t}$ perturbed by noises. A dynamic state-space form employed in the Bayesian inference framework is shown as follows [11],

$$
\begin{gathered}
\text { state transition model : } s_{t}=f_{t}\left(s_{t-1}, \epsilon_{t}\right) \\
\text { observation model : } o_{t}=h_{t}\left(s_{t}, \nu_{t}\right)
\end{gathered}
$$

where $s_{t}, o_{t}$ represent system state and observation, $\epsilon_{t}, \nu_{t}$ is the system noise and observation noise, $f_{t}(.,$.$) characterizes the kinematics of object, and h_{t}(.,$.$) models the$ observation. The key idea of Bayesian inference is to approximate the posterior probability distribution by a weighted sample set $\left\{\left(s^{(n)}, \pi^{(n)}\right) \mid n=1 \cdots N\right\}$. Each sample consists of an element $s^{(n)}$ which represents the hypothetical state of an object and a corresponding discrete sampling probability $\pi^{(n)}$, where $\sum_{n=1}^{N} \pi^{(n)}=1$. First, the sample set is resampled to avoid the degeneracy problem, and the new sample is propagated according to the state transition model. Then each element of the set is weighted with probability $\pi^{(n)}=p\left(o_{t} \mid S_{t}=s_{t}^{(n)}\right)$, which is calculated from the observation model. Finally, the state estimate $\hat{s_{t}}$ can be either be the minimum mean square error (MMSE) estimation or the maximum a posterior (MAP) estimation.

\section{Kernel-Bayesain Based Tracking Framework}

The kernel based methods enjoy a low computational complexity but often trap in local minimal/maxima, while Bayesian based methods improve robustness of the tracking process, but they suffer a large computational load by generating a huge number of hypotheses to cover the target. As a result, we propose a unified Kernel-Bayesain tracking framework to combine the merits of both methods. 


\subsection{Kernel-Bayesian Framework}

A state transition model is a basic component to be considered as the Bayesian inference is adopted for tracking. Most of the existing approaches take the naively random walk around previous system state [12] or learn through a pre-labeled video sequences [13]. The former one contains little information about the motion of the target, and thus involves a quite large computational load since many hypotheses need to be randomly generated to cover the target. While the latter one often suffers a over fitting problem, consequently available only to the training sequences. Since the mean shift algorithm provides the motion direction to the groundtruth in its iterations, which motivates us to embed the kernel method into Bayesain framework to provide a heuristic prior.

In detail, the mean shift algorithm is firstly applied to the current frame to obtain the direction of motion and the offset of the state, which are then incorporated into the transition model as prior information. In this way, the kernel based method and the Bayesian based method are combined into a unified framework. Furthermore, it is investigated [14] that symmetric kernels are amenable to mean shift iterations, which means that our framework is general to all the symmetric appearance models.

\subsection{An Optimization View}

A reinterpretation of the Kernel-Bayesian framework in an optimization view is presented to show why this framework can combine the merits of both the kernel method and the Bayesian method.

To give a clear view, an input image with three templates superimposed, corresponding to the initialization, local maximum and globe maximum is illustrated in the left column of Fig. 1, and its cost function based on our appearance model is shown in the right column of Fig. 1. As witnessed by Fig. 1. starting from the initial position, the kernel method converges to the local maximal point which is near to the global maximal point. It is clear that a few number of hypotheses generated around the local maximum point is enough to cover the the global maximal point. Otherwise, if the tracker starts from the initial position, numerous hypotheses need to be generated in order to reach the target, and the algorithm even may trap into the curse of dimensionality in the case of high-dimensionality. In our proposed framework, the deterministic optimization method is used to refine the initial position and provide a heuristic prior, and the stochastic method is then adopted to reach the globe optimal point.
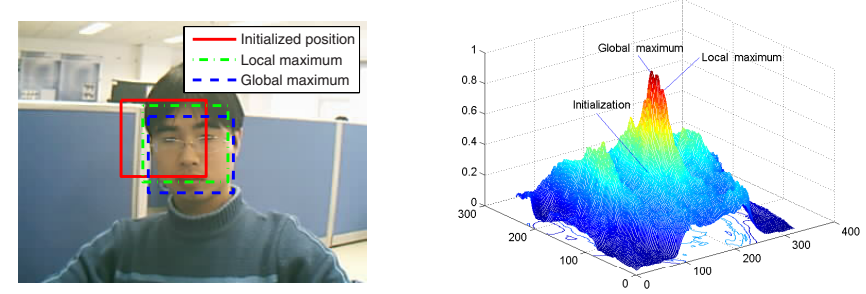

Fig. 1. (left) An input image with three templates superimposed, corresponding to the initialization (red), local maximum (green) and globe maximum (blue) and (right) its cost function 


\section{The Proposed Tracking Algorithm}

An overview of the proposed algorithm is systematically presented in Fig. 2. First a kernel based prior information is obtained through mean shift iterations, which controls both the number of the hypotheses and the directional offset of the state in the state transition model. After hypotheses precess, each hypothesis is evaluated by the spatial constraint MOG based observation model. Finally, a maximum a posterior (MAP) estimate of state is obtained based on the probability of each hypothesis. Meanwhile, a selective updating scheme is developed to update parameters of the appearance model to accommodate the changes of object and environment. Each component in this algorithm is described detailedly in the following sections.

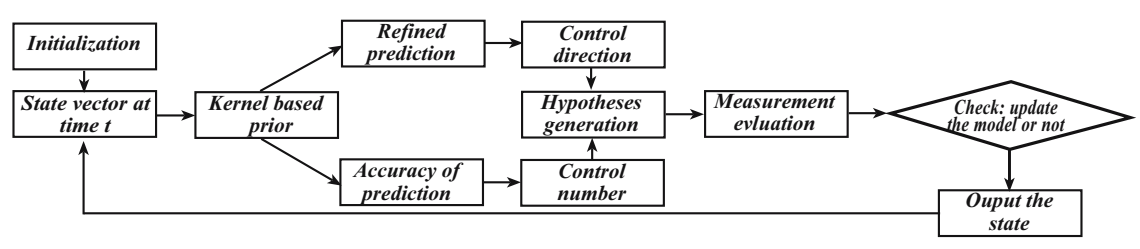

Fig. 2. The flow chart of our Kernel-Bayesian based tracking algorithm

\subsection{Spatial Constraint MOG Based Appearance Model}

The appearance of the target is modeled by a spatial constraint MOG, with the parameters estimated by an on-line EM algorithm.

Appearance Model: Similar to [4],[8], the appearance model consists of three components $S, W, F$, where $S$ component captures temporally stable images, $W$ component characterizes the two-frame variations, and $F$ component is a fixed template of the target to prevent the model from drifting away. However, this appearance model treats each pixel independently and discards the spatial outline of the target. So it may fail in the case that, for instance, there are several similar objects close to the target or partial occlusion. In our work, we apply a 2-D gaussian spatial constraint to the $S W F$ based appearance model, whose mean vector is the coordinate of the center position and the diagonal elements of the covariance matrix are proportional to the size of the target in the corresponding spatial direction, as illustrated in Fig. 3 .

As a result, the likelihood function of the spatial constraint appearance model can be formulated as follows,

$$
p\left(o_{t} \mid s_{t}\right)=\prod_{j=1}^{d}\left\{N\left(x(j) ; x_{c}, \Sigma_{c}\right) * \sum_{i=s, w, f} \pi_{i, t}(j) N\left(o_{t}(j) ; \mu_{i, t}(j), \sigma_{i, t}^{2}(j)\right)\right\}
$$

where $N\left(x ; \mu, \sigma^{2}\right)$ is a Gaussian density

$$
N\left(x ; \mu, \sigma^{2}\right)=\left(2 \pi \sigma^{2}\right)^{-1 / 2} \exp \left\{-\frac{(x-\mu)^{2}}{2 \sigma^{2}}\right\}
$$



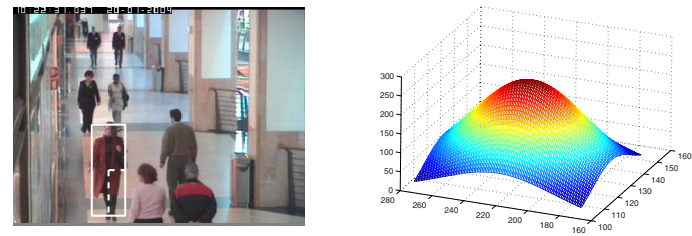

Fig. 3. A 2-D gaussian spatial constraint MOG based appearance model

and $\left\{\pi_{i, t}, \mu_{i, t}, \sigma_{i, t}, i=s, w, f\right\}$ represent mixture probabilities, mixture centers and mixture variances respectively, $d$ is the number of pixels inside the target, $x_{c}$ and $\Sigma_{c}$ represent the center of the target and its variance matrix in the spatial space.

Parameter Estimation: In order to make the model parameters dependent more heavily on the most recent observation, we assume that the previous appearance is exponentially forgotten and new information is gradually added to the appearance model. To avoid having to store all the data from previous frames, an on-line EM algorithm [4] is used to estimate the parameters as follows.

Step 1: During the E-step, the ownership probability of each component is computed as

$$
m_{i, t}(j) \propto \pi_{i, t}(j) N\left(o_{i, t}(j) ; \mu_{i, t}(j), \sigma_{i, t}^{2}(j)\right)
$$

which fulfills $\sum_{i=s, w, f} m_{i, t}=1$.

Step 2: The mixing probability of each component is estimated as

$$
\pi_{i, t+1}(j)=\alpha m_{i, t}(j)+(1-\alpha) \pi_{i, t}(j) ; i=s, w, f
$$

and a recursive form for moments $\left\{M_{k, t+1} ; k=1,2\right\}$ are evaluated as

$$
M_{k, t+1}(j)=\alpha o_{t}^{k}(j) m_{s, t}(j)+(1-\alpha) M_{k, t}(j) ; k=1,2
$$

where $\alpha=1-e^{-1 / \tau}$ acts as a forgotten factor and $\tau$ is a predefined constant.

Step 3: The mixture centers and the variances are estimated in the M-step

$$
\begin{gathered}
\mu_{s, t+1}(j)=\frac{M_{1, t+1}(j)}{\pi_{s, t+1}(j)}, \sigma_{s, t+1}^{2}=\frac{M_{2, t+1}(j)}{\pi_{s, t+1}(j)}-\mu_{s, t+1}^{2}(j) \\
\mu_{w, t+1}(j)=o_{t}(j), \sigma_{w, t+1}^{2}(j)=\sigma_{w, 1}^{2}(j) \\
\mu_{f, t+1}(j)=\mu_{f, 1}(j), \sigma_{f, t+1}^{2}(j)=\sigma_{f, 1}^{2}(j)
\end{gathered}
$$

In fact, updating of the appearance model every frame may be dangerous in case that, for instance, some backgrounds are misplaced into the target or the target is occluded. Thus, we developed a selective adaptation scheme to tackle such cases, which is described detailedly in section 4.3.

\subsection{Kernel-Bayesian Based Tracker}

As stated in the section 3, the motivation of embedding the mean shift algorithm into the Bayesian filtering framework is to provide a heuristic prediction to the state transition 
model, and thus to ease the computational burden and avoids the sample degeneracy problem.

Suppose the target is well localized at $x_{t-1}$ in frame $t-1$, we first apply mean shift iterations to the frame $t$, and the convergent position is considered as the refined initialization denoted as $\hat{x}_{t}$. In order to embed the spatial constraint appearance model into the mean shift algorithm, the weighted kernel function is defined as follows.

$$
\omega(x)=N\left(x, x_{c}, \Sigma_{c}\right) \sum_{i=w, s, f} \pi_{i, t}(j) N\left(o_{t}(x) ; \mu_{i, t}(x), \sigma_{i, t}^{2}(x)\right)
$$

And the flat kernel is chosen, so the mean shift iteration can be written as

$$
\hat{x}_{t}=\frac{\sum_{x_{i}=1}^{d} w\left(x_{i}\right) x_{i}}{\sum_{x_{i}=1}^{d} w\left(x_{i}\right)}, x_{i} \in \text { candidate. }
$$

The result obtained from mean shift iterations is then integrated into a fist-order state transition model to form an adaptive state transition model.

$$
s_{t}=\hat{s}_{t-1}+\operatorname{Affine}\left(\hat{x}_{t}-x_{t-1}\right)+\epsilon_{t}
$$

Where Affine is denoted for the affine transformation. Meanwhile, the accuracy of the refined position is evaluated by our appearance model to adaptively control the number of hypotheses and the system noise $\epsilon_{t}$. Finally the Bayesian inference is carried out based on the adaptive state transition model to achieve a robust and efficient tracking algorithm.

\subsection{Selective Adaptation for Appearance Model}

In most tracking applications, the tracker must simultaneously deal with the changes of both the target and the environment. So it is necessary to design a adaption scheme for the appearance model. However, over updating of the model may gradually introduce the noise of background into the target model, causing the model drift away finally. Thus, a proper updating scheme is of significant importance for the tracking system.

In this part, we propose a selective updating scheme based on three different confidence measures of the appearance model. First the MAP estimated state is respectively evaluated by the appearance model, the $S W$ combined components, and the $F$ component, denoted as $\pi_{a}, \pi_{s w}, \pi_{f}$. And $\left\{T_{a}, T_{s w}, T_{f}\right\}$ represent three thresholds correspondingly. Each component of the appearance model is updated selectively as follows.

It is investigated that $S$ together with $W$ components effectively capture the variations of the target and $F$ prevents the model from drifting away. As a result, such a selective updating strategy not only effectively captures the variations of the target, but also reliably prevents the drifting away problem during the tracking process.

\section{Experimental Results}

In our experiments, affine transformation is chosen to model the object motion. Specifically, the motion is characterized by $s=\left(t_{x}, t_{y}, a_{1}, a_{2}, a_{3}, a_{4}\right)$ where $\left\{t_{x}, t_{y}\right\}$ denote the 2-D translation parameters and $\left\{a_{1}, a_{2}, a_{3}, a_{4}\right\}$ are deformation parameters. Each candidate image is rectified to a $30 \times 15$ patch, and thus the feature is a 450 -dimensional vector with zero-mean-unit-variance normalization. All of the experiments are realtimely carried out on a dual-CPU Pentium IV 3.2GHz PC with 512M memory. 
Table 1. Selective Adaptation for the Appearance Model
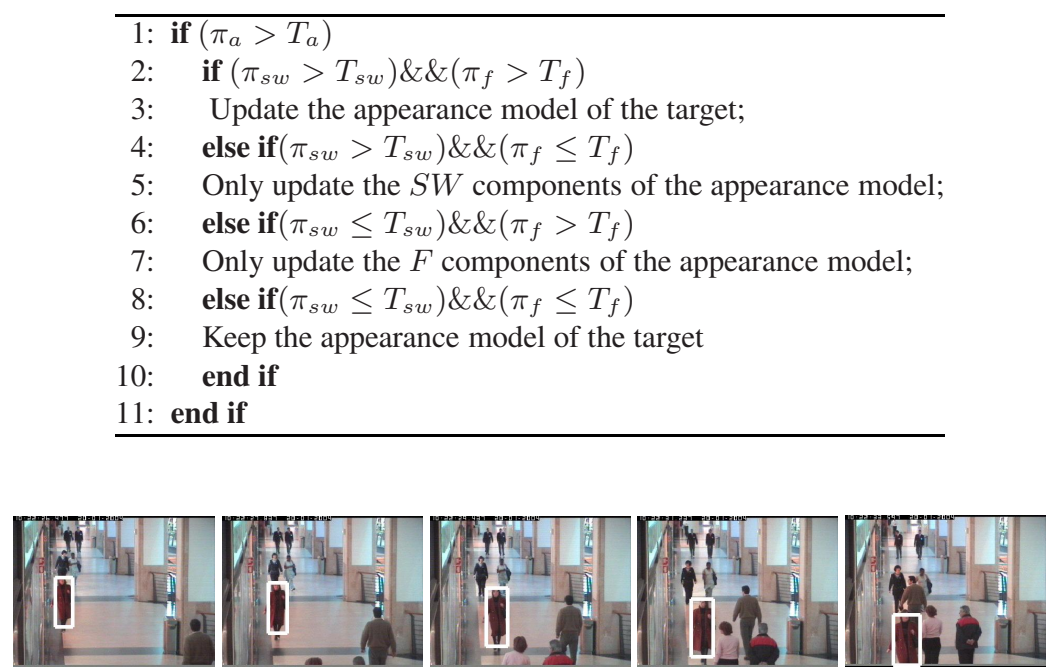

(a) Tracking performance in Kernel-Bayesian framework

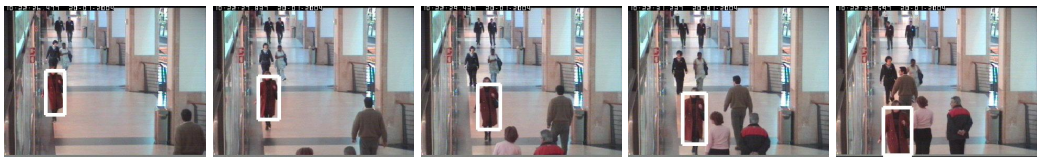

(b) Tracking performance in traditional Bayesian framework

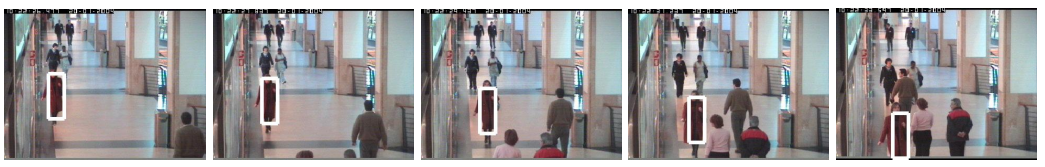

(c) Tracking performance in traditional kernel based framework

Fig. 4. Experimental performance in the different tracking frameworks

\subsection{Single Object Tracking}

In this section, three parts of experiments are presented to demonstrate the claimed contributions of the proposed tracking algorithm.

The first part shows experimental performance of our tracking framework, and a comparison to the traditional Bayesian framework and kernel based framework in both tracking accuracy and efficiency. As illustrated in Fig. 4, the first row shows the tracking performance of our algorithm, where the tracker efficiently and effectively catches the target. The second row gives the similar tracking performance in the traditional Bayesian framework with 400 hypotheses. In the third row, it is clear that the kernel method usually traps in local maximal, leading to the inaccurate localization. Furthermore, the accuracy and efficiency of these tracking frameworks are quantitatively evaluated to have a profound analysis. The tracking time with respect to the frame index is shown in the left panel of Fig 5 . And the tracking accuracy is also measured by the 

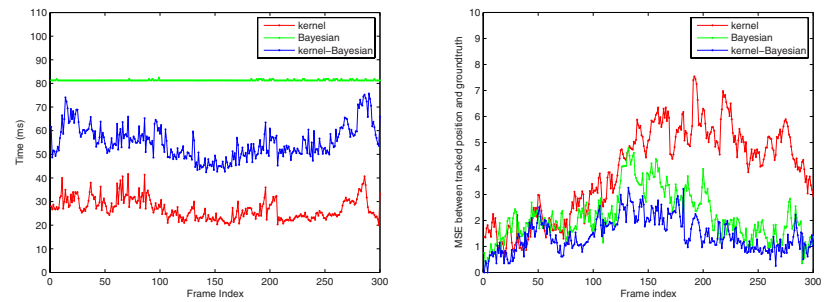

Fig. 5. (left) Tracking time with respect to the frame index, (right) MSE between estimated points and groundtruth (red: kernel, green: Bayesian, blue: kernel-Bayesian)

MSE (mean square error) between tracked position and groundtruth which is shown in the right panel of Fig 5, The results in Fig. 5] shows that the kernel method performs efficiently but has a poor performance in localization. In contrast, Bayesian based tracking algorithm achieves more accurate performance due to large number hypotheses. It possesses $81 \mathrm{~ms}$ (millisecond) tracking time in each frame and the average MSE is 8.6521. while in Kernel-Bayesian framework, the tracking time taken by each frame is only $55 \mathrm{~ms}$ on average, which greatly eases the computational burden of the Bayesian framework. The average MSE for our algorithm is only 5.8012, because the kernel method provide a heuristic prior to the state transition model which avoids the sample degeneracy suffered by the Bayesian framework and thus leads to the accurate localization.

The comparison between spatial constraint MOG based appearance model with traditional MOG based appearance model is presented in the second part. It is clear that the SMOG based appearance model gives a good solution to handle the case where there are some similar objects around the target, while the traditional MOG based appearance model fails, as shown in Fig. 6 The mechanism behind it is that the former one extracts the spatial layout of the target, which makes the model more discriminative.

The last part tests the proposed algorithm in the variational scenes. In Fig. $7 \mathrm{la}$ ), it is clear that the selective updating scheme easily absorbs the the illumination changes. Fig. 7b) shows the result of our algorithm to track a girl's head with an out-plane rotation, from which we notice that the scheme also effectively captures the variations of appearance.
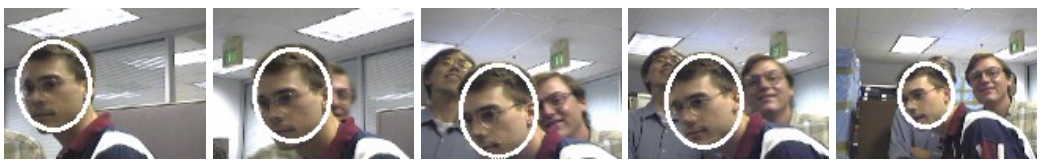

(a) Tracking with spatial constraint SWF model
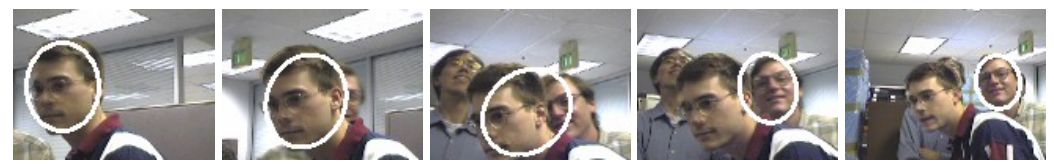

(b) Tracking with traditional SWF model

Fig. 6. Experimental results with different appearance models in the clutter scene 

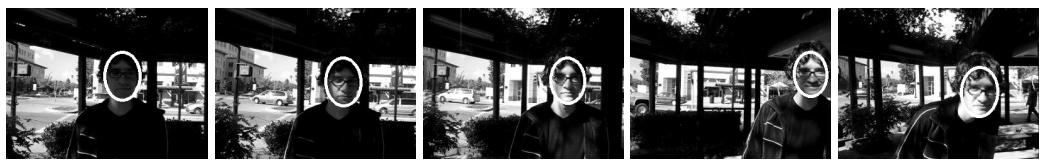

(a) Scene with large illumination changes
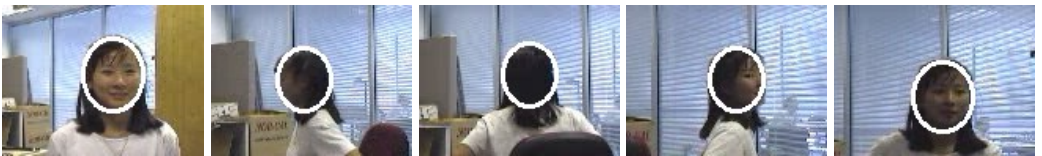

(b) Object with out-plane rotation

Fig. 7. Experimental results in different scenes (illumination change, out plane rotation)
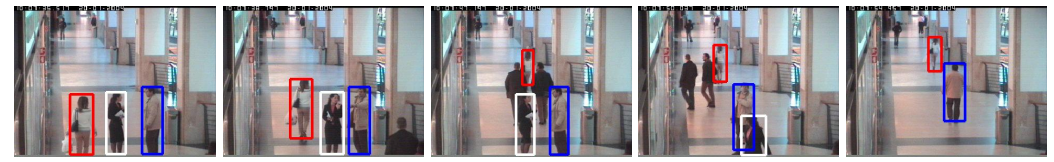

Fig. 8. Results of multiple objects tracking with the proposed tracking algorithm

\subsection{Multiple Object Tracking}

Although the major tracking task in the experiments above performs with single object, our algorithm can be easily extended to multiple object tracking.

As shown in Fig. 8, three objects are initialized manually, and can be tracked well in the following sequences including some partial occlusion cases, because the spatial constraint of appearance is employed to make the model less dependent on the peripheral pixels and the selective updating scheme effectively prevents introducing the noise into the appearance model. Due to its computational efficiency of our algorithm, it performs better and has more potential to handle various problems in the multiple object tracking than other tracking methods.

\section{Conclusion}

This paper has proposed a robust and efficient Kernel-Bayesian framework for visual tracking. In this framework, the object to be tracked is characterized by a spatial constraint MOG based appearance model, which is proved more discriminative than the traditional MOG based appearance model. Our proposed tracking framework combines the merits of both the stochastic and deterministic tracking approaches in a unified framework: the mean shift algorithm is embedded into the Bayesian framework seamlessly to give a heuristic prediction to the state transition model, aiming at effectively alleviating the great computational load and avoiding sample degeneracy suffered by the conventional Bayesian trackers. Moreover, a selective updating scheme is developed to effectively accommodate the changes in both appearance and illumination. Experimental results have demonstrated the efficiency and effectiveness of the proposed tracking algorithm. 


\section{Acknowledgment}

This work is partly supported by NSFC (Grant No. 60520120099 and 60672040) and the National 863 High-Tech R\&D Program of China (Grant No. 2006AA01Z453).

\section{References}

1. Kass, M., Witkin, A., Terzopoulos, D.: Snakes: active contour models. International Journal of Computer Vision 1(4), 321-332 (1988)

2. Isard, M., Blake, A.: Condensation: conditional density propagation for visual tracking. International Journal of Computer Vision 29(1), 5-28 (1998)

3. Comaniciu, D., Ramesh, V., Meer, P.: Kernel-based object tracking. IEEE Transactions on Pattern Analysis and Machine Intelligence 25(5), 234-240 (2003)

4. Jepson, A.D., Fleet, D.J., El-Maraghi, T.F.: Robust online appearance models for visual tracking. IEEE Transactions on Pattern Analysis and Machine Intelligence 25(10), 1296-1311 (2003)

5. Rasmussen, C., Hager, G.D.: Probabilistic Data Association Methods for Tracking Complex Visual Objects. IEEE Transactions on Pattern Analysis and Machine Intelligence 23(6), 560 $576(2001)$

6. Hager, G.D., Hager, P.N.: Efficient region tracking with parametric models of geometry andillumination. IEEE Transactions on Pattern Analysis and Machine Intelligence 20(10), 1025-1039 (1998)

7. Sullivan, J., Rittscher, J.: Guiding random particles by deterministic search. In: International Conference on Computer Vision, pp. 323-330 (2001)

8. Zhou, S., Chellappa, R., Moghaddam, B.: Visual Tracking and Recongnition Using Appearance-adaptive Models in Particles Filters. IEEE Transaction on Image Processing 13(11), 1491-1506 (2004)

9. McKenna, S., Jabri, S., Gong, S.: Tracking Colour Objects Using Adaptive Mixture Models. Image and Vision Computing 17(3-4), 225-231 (1999)

10. Fukunaga, K., Hostetler, L.: The Estimation of the Gradient of a Density Function, with Applications in Pattern Recognition. IEEE Transactions on Information Theory 21(1), 32 40 (1975)

11. Arulampalam, M., Maskell, S., Gordon, N., Clapp, T.: A tutorial on particles filters for online nonlinear/non-gaussian bayesian tracking. IEEE Transactions on Signal Processing 50(2), 174-188 (2002)

12. Yang, C.J., Duraiswami, R., Davis, L.: Fast multiple object tracking via a hierarchical particle filter. In: Proceeding of International Conference on Computer Vision, pp. 212-219 (2005)

13. Nummiaro, K., Koller-Meierand, E., Van Gool, L.: An Adaptive Color-Based Particle Filter. Image and Vision Computing 21(1), 99-110 (2003)

14. Parameswaran, V., Ramesh, V., Zoghlami, I.: Tunable Kernels for Tracking. In: Proceeding of International Conference on Computer Vision and Pattern Recognition, pp. 2179-2186 (2006) 